\section{(C) OPEN ACCESS}

\title{
Multicentre, randomised controlled trial of PDSAFE, a physiotherapist-delivered fall prevention programme for people with Parkinson's
}

\author{
Kim Chivers Seymour, ${ }^{\oplus}$ Ruth Pickering, ${ }^{2}$ Lynn Rochester, ${ }^{3}$ Helen C Roberts, ${ }^{2}$ \\ Claire Ballinger, ${ }^{2}$ Sophia Hulbert, ${ }^{1}$ Dorit Kunkel, ${ }^{1}$ Ioana R Marian, ${ }^{2}$ Carolyn Fitton, ${ }^{1}$ \\ Emma Mclntosh, ${ }^{4}$ Victoria A Goodwin, ${ }^{\circ}$ Alice Nieuwboer, ${ }^{6}$ Sarah E Lamb, ${ }^{7}$ \\ Ann Ashburn ${ }^{\circ}$
}

\begin{abstract}
- Additional material is published online only. To view please visit the journal online (http://dx.doi.org/10.1136/ jnnp-2018-319448).
\end{abstract}

${ }^{1}$ School of Health Sciences, University of Southampton, Southampton, UK

${ }^{2}$ Faculty of Medicine, University of Southampton, Southampton, UK

${ }^{3}$ Institute of Neuroscience, Newcastle University, Newcastle upon Tyne, United Kingdom ${ }^{4}$ HEHTA, University of Glasgow, Glasgow, UK

${ }^{5}$ College of Medicine, University of Exeter, Exeter, UK

${ }^{6}$ Department of Rehabilitation Sciences, KU Leuven, Leuven, Belgium

Oxford Clinical Trials Research Unit, University of Oxford, Oxford, UK

Correspondence to Professor Ann Ashburn, School of Health Sciences, University of Southampton, Southampton S017 1BJ, UK; ann@soton.ac.uk

Received 14 August 2018 Revised 6 February 2019 Accepted 9 February 2019 Published Online First 3 April 2019

Check for updates

(c) Author(s) (or their employer(s)) 2019. Re-use permitted under CC BY-NC. No commercial re-use. See rights and permissions. Published by BMJ.

To cite: Chivers Seymour K, Pickering $R$, Rochester $L$, et al. I Neurol Neurosurg Psychiatry 2019:90:774-782.

\section{ABSTRACT \\ Objective To estimate the effect of a physiotherapist- delivered fall prevention programme for people with Parkinson's (PwP).}

Methods People at risk of falls with confirmed Parkinson's were recruited to this multicentre, pragmatic, investigator blind, individually randomised controlled trial with prespecified subgroup analyses. 474 PwP (Hoehn and Yahr 1-4) were randomised: 238 allocated to a physiotherapy programme and 236 to control. All participants had routine care; the control group received a DVD about Parkinson's and single advice session at trial completion. The intervention group (PDSAFE) had an individually tailored, progressive home-based fall avoidance strategy training programme with balance and strengthening exercises. The primary outcome was risk of repeat falling, collected by self-report monthly diaries, 0-6 months after randomisation. Secondary outcomes included Mini-BESTest for balance, chair stand test, falls efficacy, freezing of gait, health-related quality of life (EuroQol EQ-5D), Geriatric Depression Scale, Physical Activity Scale for the Elderly and Parkinson's Disease Questionnaire, fractures and rate of near falling.

Results Average age is 72 years and 266 (56\%) were men. By 6 months, 116 (55\%) of the control group and $125(61.5 \%)$ of the intervention group reported repeat falls (controlled OR 1.21, 95\% Cl 0.74 to 1.98, $\mathrm{p}=0.447)$. Secondary subgroup analyses suggested a different response to the intervention between moderate and severe disease severity groups. Balance, falls efficacy and chair stand time improved with near falls reduced in the intervention arm.

Conclusion PDSAFE did not reduce falling in this pragmatic trial of PwP. Other functional tasks improved and reduced fall rates were apparent among those with moderate disease.

Trial registration number ISRCTN48152791.

\section{INTRODUCTION}

Parkinson's disease (PD) is a progressive neurological condition that affects more than 10 million people worldwide; falls are common in this group. Prior to the start of the PDSAFE trial, a meta-analysis of fall reduction among the general older population ${ }^{1}$ demonstrated a positive exercise effect but it was not until during PDSAFE that a meta-analysis of fall prevention among people with Parkinson's (PwP) was published ${ }^{2}$; findings were inconsistent with small overall effect sizes on fall rate ratios (FRR) and group-based training more favourable than home-based interventions. ${ }^{3-8}$

Falls are twice as common among PwP as the healthy aged. ${ }^{17-10}$ Impairments of gait, including freezing of gait, cognition and loss of postural control, are common risk factors for falling in this population, balance and falls are not easily remedied by medication. ${ }^{11} 12$ The effects of falls in PwP can be profound including loss of mobility, poor function in activities of daily living, fractures and increased care needs ${ }^{7-9} 1213$ as well as being costly to both individuals and health services. ${ }^{14}$ There is some evidence that exercise may be useful for fall prevention in PwP. $^{2}$ However, trials conducted to date have usually been small, only 6 out of 25 trials in the meta-analysis recruited 100 or more participants. Most of the larger trials were negative ${ }^{2}$ but there were differences in subgroup responses ${ }^{6-8}$; benefits were demonstrated in higher functioning groups, particularly in people who had sufficient function to enable them to travel to exercise venues and participate in group programmes. ${ }^{6}$ Canning $e t a l^{6}$ recruited $231 \mathrm{PwP}$ to a randomised controlled trial (RCT) and reported non-significant overall effect but significant interaction with disease severity; those with more severe disease had a greater number of falls following intervention, while there was significant improvement for those with less severe disease.

Our aim was to compare the effect on repeat falling outcomes and other outcomes of an individualised, progressive exercise intervention delivered in people's own home, in addition to usual care. We aimed to recruit a large, pragmatic sample of PwP, and used prespecified and exploratory subgroup analysis to examine variation in the intervention effect according to baseline disease severity and freezing of gait.

\section{METHODS}

Study design

A full protocol has been published. ${ }^{15}$ This was a multicentre, pragmatic, investigator-masked RCT. Participants were recruited from Parkinson's 
services in National Health Service hospitals and clinics, community and social services in eight regions across England: Bournemouth and Poole, Truro, Plymouth, Exeter, Winchester and Basingstoke, Newcastle, Portsmouth and Southampton. We also identified recruits via the study website. We delivered interventions in the participant's home. The primary endpoint for the trial was 6 months after randomisation. We received funding to allow 12-month follow-up on the first 342 recruited into the trial. An economic evaluation and a nested qualitative study of the views of participants will be published elsewhere as will details of the intervention. Trial management, data monitoring and statistical analyses were coordinated at the PDSAFE trial office, University of Southampton, UK and supported by the Oxford Clinical Trials Research Unit (OCTRU).

\section{Participants}

People were eligible if they had a clinically confirmed diagnosis of Parkinson's disease in accordance with UK Brain Bank criteria ${ }^{16}$; were living in their own home; independently mobile with or without an aid; experienced at least one fall in the previous 12 months; scored 24 or more on Mini-Mental State Examination ${ }^{17}$ (MMSE); had the cognitive ability to give informed consent; were able to understand and follow commands; and considered able to participate in an exercise and strategy programme.

\section{Interventions}

All participants continued to receive usual care including medication management, and took part in their usual activities, such as exercise or social groups. Participants assigned to the control group were given a Parkinson's UK DVD with information about Parkinson's. At the end of their time in the trial, control participants received a single session on fall avoidance and a booklet on falls management.

PDSAFE $^{18}$ comprised individually tailored, progressive homebased exercise and strategies to avoid falls, for example, 'Stepping patterns and sideways stepping in small complex areas to avoid tripping'. Registered physiotherapists made visits to the participants' home to provide 12 supervised sessions of 1-1.5 hours duration over 6 months. More supervised sessions were delivered early in the programme, and gradually tapered over time. From the start of the programme, participants were expected to engage in daily unsupervised exercise for about $30 \mathrm{~min}$, and were provided with a prescription for these and strategies to maximise adherence, such as integration into daily routines. Participants were given a folder with pictures and descriptions of exercises, a rating of perceived exertion scale, an exercise log, and DVDs of both exercise demonstrations and personal videos taken by their physiotherapist of them doing the exercises. Programmes were based on an individualised assessment of fall risk factors, including the home environment and high-risk activities. Exercises included balance training with a gradual decrease in the area of support and use of foam standing pads. Strength training used body weight resistance, and if appropriate, weighted vests, during functional exercises (sit to stand). Strategies for fall and freezing avoidance included transfer practice (turning) in complex environments such as bathrooms and kitchen and adopting wide-based positions during functional task practice.

Intervention fidelity was promoted through a 2-day training session, and subsequent observation and feedback during sessions by a specialist physiotherapist with expertise in Parkinson's management. All but one physiotherapist had a background in neurological practice. Monthly 'master classes', weekly teleconferences and regular clinical supervision sessions were implemented. Fidelity checks were undertaken three times during a therapist's first 3 months of delivering the intervention, and three times monthly thereafter. We considered that people who attended the assessment and received one home session to have engaged with the intervention. Those who had seven or more sessions were considered to have complied with the intervention.

\section{Outcomes}

The primary outcome was risk of repeat falling in the first 6 months after randomisation. A fall was defined as an event which resulted in a person coming to rest unintentionally on the ground or lower level. ${ }^{19}$ Secondary outcomes were fractures and the rate of near falling; the Mini-BESTest ${ }^{20}$ (high valuesgood, a test of balance control); the chair stand test (CST) ${ }^{21}$ (low timing-good); medication use; the Geriatric Depression Scale (GDS) $^{22}$ (15 questions version; low scores-good); the International version of the Falls Efficacy Scale (FES) ${ }^{23}$ (low scoresgood); the New Freezing of Gait Questionnaire (NFoG) ${ }^{24}$ (low scores-good); the Parkinson's Disease Questionnaire (PDQ$39)^{25}$ (low scores-good, a quality of life measure designed specifically for PwP); the Physical Activity Scale for the Elderly (PASE) $^{26}$ (high scores-good) at 6 months; and the EuroQol (EQ-5D-3L) health-related quality of life instrument. ${ }^{27} \mathrm{We}$ recorded all deaths, any hospitalisations were recorded at assessment and serious adverse events were reviewed by a medical physician (HR). The PASE, PDQ-39 and GDS were self-completed and returned by post.

\section{Randomisation and blinding}

Prior to randomisation, participants completed 3 months of fall diaries. We then did a baseline assessment after which participants were randomly assigned (50:50) to either the intervention or control group, using an online procedure set up by OCTRU (a UK Clinical Research Collaboration registered trial unit). The allocations were sent to the trial manager who informed a treating therapist, to ensure allocation concealment from trial recruiters and assessors. Random allocations were computer generated, stratified by centre and allocated in blocks with random size of $2,4,6$ or 8 . The trial manager, the treating therapists and the participants were not blinded to the allocated group. At each point of contact, participants were asked not to divulge their group allocation to trial assessors who were blind to group allocation.

\section{Procedure}

We trained registered healthcare professionals to assess eligibility of potential participants, to register and randomise participants, to collect baseline information and administer outcome measures during the follow-up period; fidelity checks were routinely made. We collected eligibility and baseline data over two visits, separated by a 3 -month period in which we asked participants to collect falls diary data. At the first eligibility visit we collected the Montreal Cognitive Assessment (MoCA), ${ }^{28}$ the number of falls experienced over the previous 12 months, the MMSE, ${ }^{17}$ the Hoehn and Yahr Scale of disease severity, ${ }^{29}$ demographics and medical history. At the second visit, we rechecked consent, collected the Unified Parkinson's Disease Rating Scale (UPDRS) ${ }^{30}$ (low scores-good, motor section only) and enrolled and randomised the participant. Participants were asked to complete monthly diaries through the follow-up period and face-to-face assessments were taken to collect all other data 
during home visits at 6 months, and for those completing the extended follow-up at 12 months.

\section{Sample size calculation}

Based on the results of a previous trial ${ }^{8}$ we assumed the risk of repeat falling between 0 and 6 to be $50 \%$ in the intervention group and $63 \%$ in the control group, yielding a sample size requirement of 228 participants per group at $80 \%$ power in a two-sided 5\% comparison of proportions. Allowing for $15 \%$ (10\% before randomisation and 5\% after) loss to follow-up, for these participants, between approach, randomisation and 6 months, we aimed to recruit 534 participants to the prerandomisation falls collection period.

\section{Statistical analysis}

A statistical analysis plan was finalised before the data set was locked. The analysis was intention to treat in that all participants were analysed in the group to which they were randomised. For the primary outcome, we included participants who had completed a minimum of $50 \%$ of diaries (as recommended by Goodwin $e t a l^{7}$ ) and missing falls diary data, for these participants, by imputing the binary indicator of repeat faller (yes/no) from the final assessment if these data were available. The analysis of rates of falling included all participants with any degree of diary completion.

We used logistic regression controlling for site, age, gender, Hoehn and Yahr, the logarithm of retrospectively collected number of falls in the year prior to screening, repeat fall status prior screening, and the logarithm of the prospectively collected rate of falling during the 3 months period prior to randomisation with 0.5 added to numerators so that participants with zero falls during the period were included. These controlling variables were finalised in a blind analysis without access to the group indicator, as specified in the analysis plan. Rates of falling during the periods $0-6$ and 6-12 months after randomisation were examined in negative binomial models with the logarithm of a participant's days of diary follow-up included as an offset. ORs and FRRs are presented with 95\% CIs. The geometric mean of individual participant rates (with 0.5 added to the numerator as before) of falling during 6-month follow-up periods is presented as rates per 6 months to accommodate the skewed distribution across participants. We prespecified subgroup analysis for falling between 0 and 6 months to $\operatorname{MoCA}^{28}$ status $(\leq 25$ and $\geq 26$ ), freezing status, and disease severity UPDRS ${ }^{30}$ and Hoehn and Yahr. ${ }^{29}$ In addition, and informed by the findings of Morris et $a l,{ }^{35}$ we explored subgroup analyses for MoCA, UPDRS and number of falls in the year prior to screening using the tertiles of the baseline distribution. We tested for subgroup effects using statistical tests of interaction. A secondary per protocol analysis excluded participants from the PDSAFE group who received less than 7 of the planned 12 sessions. Analysis was conducted in SPSS version 24 (IBM) and Stata. ${ }^{31}$

\section{Patient involvement}

Patient and Public Involvement (PPI) representatives were involved in the design of the study, the development of participant information sheets, consent forms and intervention resources. A PPI representative was a member of the Trial Steering Committee. The trial was discussed at several local Parkinson's support groups and representatives were invited to a meeting on interpretation of results and key messages.

\section{RESULTS}

\section{Trial progression and recruitment}

Recruitment was from July 2014 to August 2016 (study flow summarised in figure 1). A total of $640 \mathrm{PwP}$ were invited to participate, 99 either did not respond or did not meet the eligibility criteria leaving 541 for consent and completion of the first eligibility visit. At this point, a further seven people did not reach the MMSE requirement and were excluded. The remainder went on to second eligibility check after which a further 60 people were excluded (reasons provided in figure 1). The remaining 474 completed a baseline assessment and were randomised to usual care $(n=236)$ or PDSAFE $(n=238)$. Withdrawal rates were low at the primary time point, and equivalent across the groups. Falls diary completion was good at the primary time point, with $203 / 238(85 \%)$ of the usual care group and 211/236 (89\%) of the intervention group providing useable data, see online supplementary table 1 . The IQR for the number of diary completion days over the primary 6-month period was 174-183 and 153-183 days in the control and intervention groups, respectively. Fourteen trained assessors completed blinded data collection.

\section{Intervention}

Eighteen physiotherapists delivered PDSAFE and compliance with the intervention was good. Two hundred and seventeen of 238 (91\%) participants received per protocol seven or more supervised treatment sessions and 75\% (162) of those received the maximum number. The median number of supervised sessions for all participants randomised to the intervention was 12 (IQR 11-12). The majority of participants, 236/238, received the exercise assessment and at least one supervised session. Two participants did not start due to a change of mind, 19 received less than seven sessions. Reasons for withdrawal from the trial included: admission to a nursing home, deteriorating health, commitment was too much, no reason given and caring for others (see figure 1).

\section{Sample}

The baseline characteristics of randomised groups were balanced (table 1). There was a difference in the rate of falling in the run-in period.

\section{Outcomes}

There was little difference in the rate of falling between groups in the first 6 months (table 2). Table 3 shows the treatment effect estimates for the primary time point including the primary outcome. Repeat falling in the PDSAFE group at 6 months was slightly higher than in the control group (OR 1.21, 95\% CI 0.74 to $1.98, \mathrm{p}=0.447)$. There was significantly lower risk of near falling $(0.67,95 \%$ CI 0.53 to $0.86, p=0.001)$ in the PDSAFE compared with the control group.

For the secondary outcomes collected at 6 months (table 3), the exercise group had better balance (between-group difference Mini-BESTest score 0.95 points, 95\% CI 0.24 to $1.67, \mathrm{p}=0.009$ ); better falls confidence (between-group difference 1.60 points, 95\% CI 3.00 to $0.19, \mathrm{p}=0.026$ ); and improved balance and functional strength as assessed by the CST $(p=0.041)$ (table 4). No material differences from those reported above were found when analyses were repeated on a per protocol basis.

\section{Prespecified and exploratory subgroup analysis: 6 months}

The results of prespecified subgroup analyses for MoCA and freezing of gait are shown in figure 2, along with further analysis of subgroups defined by tertiles. People who had freezing 


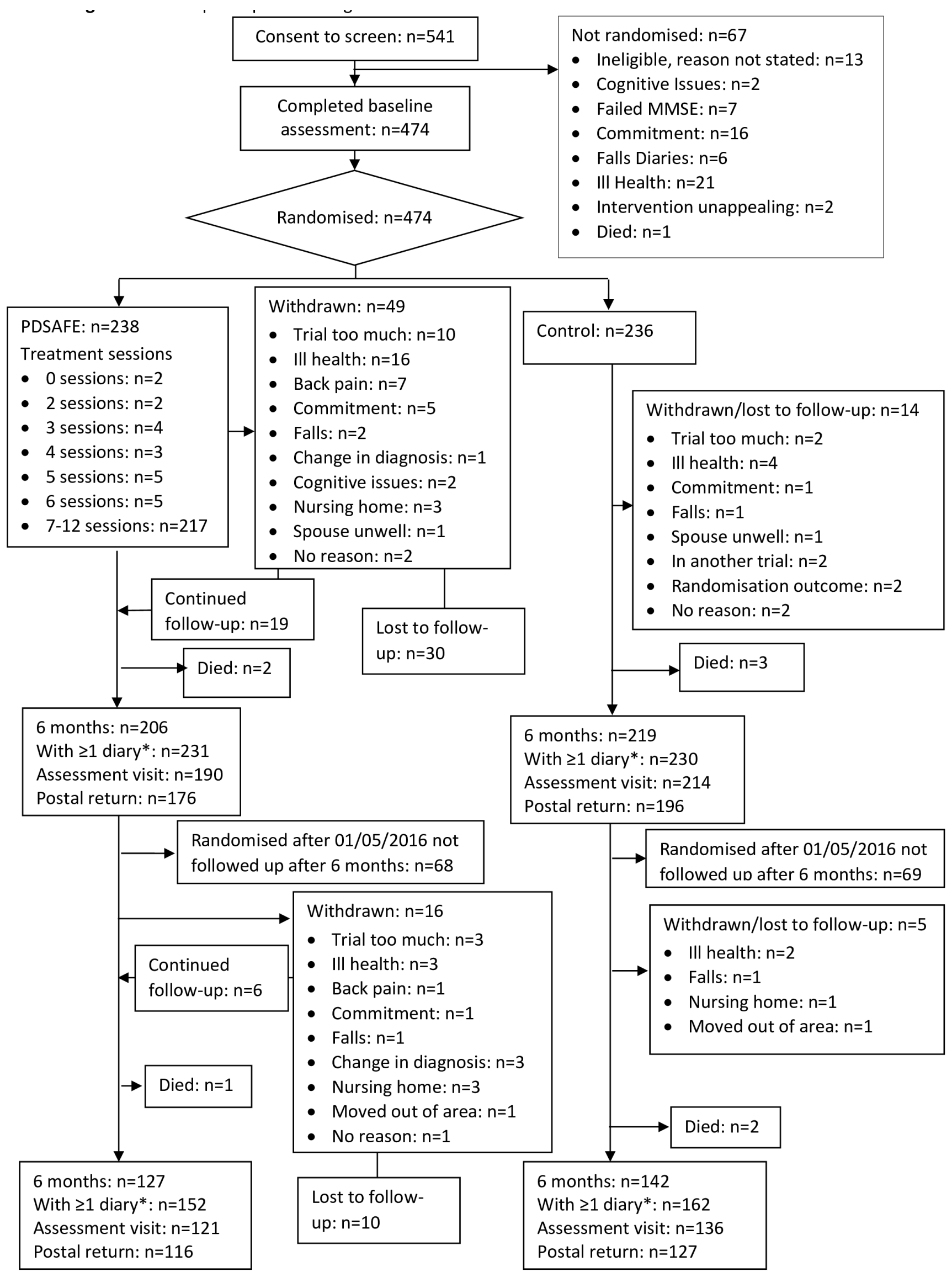

*Number with any diaries during the preceding 6 months

Figure 1 Flow of participants through the trial. MMSE, Mini-Mental State Examination. 
Table 1 Baseline characteristics in the PDSAFE and control groups: figures are number (\%) unless stated otherwise

\begin{tabular}{|c|c|c|}
\hline & $\begin{array}{l}\text { PDSAFE } \\
\left(n=238^{*}\right)\end{array}$ & $\begin{array}{l}\text { Control } \\
(n=236 t)\end{array}$ \\
\hline \multicolumn{3}{|l|}{ Gender } \\
\hline Male & $147(62 \%)$ & $119(50 \%)$ \\
\hline Female & $91(38 \%)$ & $117(50 \%)$ \\
\hline \multicolumn{3}{|l|}{ Age (years) } \\
\hline Mean (SD) & $71(7.7)$ & $73(7.7)$ \\
\hline Min to $\max$ & 51 to 91 & 46 to 88 \\
\hline \multicolumn{3}{|l|}{ Disease duration (years) } \\
\hline Mean (SD) & $8(6.6)$ & $8(5.8)$ \\
\hline Min to $\max$ & 0 to 36 & 0 to 29 \\
\hline \multicolumn{3}{|l|}{ MMSE } \\
\hline Mean (SD) & $28(1.7)$ & $29(1.6)$ \\
\hline Min to $\max$ & 24 to 30 & 24 to 30 \\
\hline \multicolumn{3}{|l|}{ MoCA } \\
\hline Mean (SD) & $26(2.9)$ & $26(3.2)$ \\
\hline Min to $\max$ & 15 to 30 & 9 to 30 \\
\hline$\leq 25$ (cognitively impaired) & $91(38 \%)$ & $93(39 \%)$ \\
\hline \multicolumn{3}{|l|}{ Living status } \\
\hline Lived alone & $48(20 \%)$ & $59(25 \%)$ \\
\hline With a spouse/partner & $174(73 \%)$ & $166(70 \%)$ \\
\hline With a friend/family & $15(6 \%)$ & $10(4 \%)$ \\
\hline \multicolumn{3}{|l|}{ Hoehn and Yahr stage } \\
\hline 1 & $26(11 \%)$ & $30(13 \%)$ \\
\hline 2 & $78(33 \%)$ & $56(24 \%)$ \\
\hline 3 & $102(43 \%)$ & $112(48 \%)$ \\
\hline 4 & $32(13 \%)$ & $38(16 \%)$ \\
\hline \multicolumn{3}{|l|}{ UPDRS } \\
\hline Mean (SD) & $32(15.2)$ & $33(17.3)$ \\
\hline Min to $\max$ & 2 to 77 & 4 to 92 \\
\hline TD phenotype & $21(9 \%)$ & $19(8 \%)$ \\
\hline PIGD phenotype & $194(83 \%)$ & $206(88 \%)$ \\
\hline Indeterminate phenotype & $20(8 \%)$ & $10(4 \%)$ \\
\hline $\begin{array}{l}\text { Freezing of gait in the past } \\
\text { month }\end{array}$ & $152(64 \%)$ & $139(59 \%)$ \\
\hline
\end{tabular}

Number of falls in 12 months prior to screening

$\begin{array}{lll}\text { Median (min to max) } & 3(1 \text { to } 1460) & 3(1 \text { to } 1095) \\ \text { Mean (SD) } & 26(132.7) & 19(105.4) \\ \text { Repeat falling in } 12 \text { months } & 186(78 \%) & 189(80 \%)\end{array}$

Rate of falls/person/3 months prior to randomisation

\begin{tabular}{|c|c|c|}
\hline Median (min to max) & 1.98 (0 to 319$)$ & 0.99 (0 to 73$)$ \\
\hline Mean (SD) & $5.9(22.8)$ & $3.0(7.3)$ \\
\hline \multicolumn{3}{|c|}{ Rate of near falls/person $/ 3$ months prior to randomisation } \\
\hline Median (min to max) & $4.4(0$ to 440$)$ & $4.3(0$ to 601$)$ \\
\hline Mean (SD) & $13.8(35.8)$ & $15.6(51.4)$ \\
\hline \multicolumn{3}{|l|}{ Medications } \\
\hline Levodopa & $208(88 \%)$ & $216(92 \%)$ \\
\hline Dopamine agonist & $108(46 \%)$ & $106(45 \%)$ \\
\hline Monoamine oxidase & $52(22 \%)$ & $46(20 \%)$ \\
\hline inhibitor & $59(25 \%)$ & $41(17 \%)$ \\
\hline COMT inhibitors & $19(8 \%)$ & $23(10 \%)$ \\
\hline Other PD medication & & \\
\hline \multicolumn{3}{|l|}{ GDS score at baseline } \\
\hline$>5$ (suggestive of depression) & $147 / 235(63 \%)$ & $164 / 236(70 \%)$ \\
\hline$\geq 10$ (indicative of depression) & $50 / 235(21 \%)$ & $49 / 236(21 \%)$ \\
\hline \multicolumn{3}{|l|}{ Coexisting conditions } \\
\hline Orthopaedic & $109(46 \%)$ & $129(54 \%)$ \\
\hline Cardio/respiratory & $85(36 \%)$ & $96(41 \%)$ \\
\hline
\end{tabular}

Continued
Table 1 Continued

$\begin{array}{ll}\text { PDSAFE } & \text { Control } \\ \left(\mathrm{n}=238^{*}\right) & (\mathrm{n}=236 \mathrm{t})\end{array}$

*Missing values in the intervention group: living status (1); UPDRS (1); disease duration (1); freezing of gait (1); rate of falling in the 3 months prior to randomisation (1); TD/PIGD/Indeterminate phenotype (3).

tMissing values in the control group: living status (1); UPDRS (1); TD/PIGD/ Indeterminate phenotype (1).

GDS, Geriatric Depression Scale; MMSE, Mini-Mental State Examination; MoCA, Montreal Cognitive Assessment; PD, Parkinson's disease; UPDRS, Unified Parkinson's Disease Rating Scale.

of gait at study entry did not respond well to the intervention, and this interaction effect estimate was statistically significant $(p=0.025)$. There were trends towards worse response to treatment in people with cognitive impairment (as measured by the MoCA) ( $p=0.111$ and $p=0.088$ for the analyses of repeat falls and rate of falling, respectively).

Statistically significant differences in the PDSAFE effect in relation to rate of falling were found across subgroups according to UPDRS severity, and retrospectively collected falling in the year prior to screening (interaction $\mathrm{p}=0.009$ and 0.050 , respectively); with an indication of possible benefit of PDSAFE in the middle subgroups only, while PDSAFE was associated with increased rate of falling in the most severe group. The impact of PDSAFE on near falling, Mini-BESTest and falls confidence was consistently beneficial across the subgroups. Additional exploratory subgroup analyses are shown in supplementary table 2 .

\section{Adverse events and deaths}

Two participants died during follow-up in the PDSAFE group and five in the control group (figure 1). During the 6 months following randomisation, five fractures were reported by PDSAFE and nine by control group participants. Nine PDSAFE and 20 control group participants reported hospitalisations, of whom one PDSAFE participant reported two stays, none of these were associated with participation in the intervention.

\section{Extended follow up}

During the period 6-12 months after randomisation, seven fractures were reported by PDSAFE and three by control group participants; 18 PDSAFE and 21 control group participants reported hospitalisations of whom two PDSAFE and four control participants reported two hospital stays. No participant fell during exercising.

\section{DISCUSSION}

PDSAFE is the largest trial of physiotherapy for fall prevention among PwP, with 474 participants randomised it was more than double the size of the previous largest trial by Canning $e \mathrm{al}^{6}$ with 231 participants. It was novel with a personalised intervention conducted in the home setting, and both a 3-month prerandomisation baseline and follow-up counts of falls collected prospectively in diaries. Despite this, we failed to demonstrate PDSAFE was effective in reducing falls in a heterogeneous sample of PwP overall. Our non-significant result was similar to that found by previous researchers of trials with more than 100 participants ${ }^{5-8}$ and falls as a primary outcome. The exception was a trial of exercises conducted in an outpatient setting by Morris et al in 2015. ${ }^{3}$ This positive outcome was not replicated when repeated at home with a lower treatment dose. ${ }^{5}$ Two other research groups reported fall reduction as a result of interventions outside of current physiotherapy; virtual reality and treadmill training ${ }^{4}$ and 
Table 2 Intention-to-treat analysis of falling outcomes: $0-6$ and 6-12 months

\begin{tabular}{|c|c|c|c|c|c|}
\hline \multirow[b]{2}{*}{ Period } & & PDSAFE & Control & \multirow{2}{*}{$\begin{array}{l}\text { PDSAFE/Control* } \\
\text { OR }(95 \% \mathrm{CI})\end{array}$} & \multirow[b]{2}{*}{ P value* } \\
\hline & & \multicolumn{2}{|l|}{ n (\%) } & & \\
\hline \multirow{6}{*}{$\begin{array}{l}\text { Repeat falling restricted to } \\
\geq 50 \% \text { diaries }\end{array}$} & Baseline & $127 / 231(55)$ & $92 / 230(40)$ & & \\
\hline & Baselinet & $112 / 203(55)$ & $80 / 211(38)$ & & \\
\hline & 0-6 months & $125 / 203(62)$ & $116 / 211(55)$ & $1.21(0.74$ to 1.98$)$ & 0.447 \\
\hline & Baselinet & $55 / 114(48)$ & $47 / 132(36)$ & & \\
\hline & 6-12 months & $57 / 114(50)$ & $71 / 132(54)$ & $0.86(0.45$ to 1.65$)$ & 0.657 \\
\hline & & \multicolumn{2}{|c|}{ Falls/person/6 months $\ddagger$} & FRR $(95 \% \mathrm{Cl})$ & \\
\hline \multirow[t]{4}{*}{ Fall rates } & Baseline & 4.5 & 3.3 & & \\
\hline & 0-6 months & 3.4 & 2.7 & $0.98(0.80$ to 1.19$)$ & 0.824 \\
\hline & 6-12 months & 2.7 & 2.8 & $0.83(0.62$ to 1.11$)$ & 0.200 \\
\hline & & \multicolumn{2}{|c|}{ Near falls/person/6 months $\ddagger$} & NFRR $(95 \% \mathrm{Cl})$ & \\
\hline \multirow[t]{3}{*}{ Near fall rates } & Baseline & 8.0 & 8.1 & & \\
\hline & 0-6 months & 4.7 & 5.6 & 0.67 (0.53 to 0.86$)$ & 0.001 \\
\hline & 6-12 months & 3.9 & 3.7 & 1.01 (0.67 to 1.52$)$ & 0.968 \\
\hline
\end{tabular}

*Controlled for site, age, gender, repeat falling or not in the year prior to screening, log number of falls in the year prior to screening, log rate of falling in the prerandomisation falls collection period and Hoehn and Yahr (NFRR additionally controlled for log rate of near falling in the prerandomisation falls collection period).

†Baseline repeat falling restricted to participants including in the 0-6 and 6-12 month analyses.

$\ddagger$ Geometric mean of individual fall rates with 0.5 added to all numerators.

FRR, fall rate ratio; NFRR, near fall rate ratio.

Table 3 Secondary outcomes at 6 and 12 months

\begin{tabular}{|c|c|c|c|c|c|}
\hline \multirow[b]{2}{*}{ Visit } & & \multicolumn{2}{|l|}{ Mean (SD, n) } & \multirow[b]{2}{*}{$\begin{array}{l}\text { Mean difference (PDSAFE- } \\
\text { Control) }(95 \% \mathrm{Cl})^{*}\end{array}$} & \multirow[b]{2}{*}{ P value* } \\
\hline & & $\begin{array}{l}\text { PDSAFE } \\
(n=238)\end{array}$ & $\begin{array}{l}\text { Control } \\
(n=236)\end{array}$ & & \\
\hline \multirow{6}{*}{$\begin{array}{l}\text { Mini-BESTest } \\
\text { (0-28, lower values worse) }\end{array}$} & tBaseline & $18.3(5.7, n=183)$ & $17.3(6.1, n=211)$ & $0.95(0.24$ to 1.67$)$ & 0.009 \\
\hline & 6 months & $19.4(5.9, n=183)$ & $17.5(6.4, n=211)$ & & \\
\hline & 6 months-baseline & $1.1(3.8, n=183)$ & $0.2(3.8, n=211)$ & & \\
\hline & $\dagger *$ Baseline & $18.5(5.8, n=115)$ & $17.5(6.1, n=126)$ & $-0.41(-1.48$ to 0.66$)$ & 0.449 \\
\hline & 12 months & $17.9(6.5, n=115)$ & $17.4(6.7, n=126)$ & & \\
\hline & 12 months-baseline & $-0.7(4.5, \mathrm{n}=115)$ & $-0.2(3.8, n=126)$ & & \\
\hline \multirow{6}{*}{$\begin{array}{l}\text { FES-I } \\
\text { (16-64, higher values worse) }\end{array}$} & †Baseline & $34.1(11.0, n=189)$ & $35.1(11.5, n=211)$ & $-1.6(-3.0$ to -0.19$)$ & 0.026 \\
\hline & 6 months & $33.4(10.6, \mathrm{n}=189)$ & $36.2(11.4, n=211)$ & & \\
\hline & 6 months-baseline & $-0.7(7.9, n=189)$ & $1.1(7.2, n=211)$ & & \\
\hline & t*Baseline & $33.4(10.7, \mathrm{n}=119)$ & $33.7(11.3, \mathrm{n}=135)$ & $-1.4(-3.41$ to 0.66$)$ & 0.184 \\
\hline & 12 months & $34.8(11.2, n=119)$ & $37.2(11.6, n=135)$ & & \\
\hline & 12 months-baseline & $1.3(8.2, n=119)$ & $3.5(9.3, n=135)$ & & \\
\hline \multirow{6}{*}{$\begin{array}{l}\text { PASE } \\
\text { (0-400, lower values worse) }\end{array}$} & †Baseline & $107.8(73.5, n=153)$ & $100.1(67.1, n=177)$ & $-1.05(-11.3$ to 9.21$)$ & 0.841 \\
\hline & 6 months & $110.2(70.4, n=153)$ & $100.6(68.0, n=177)$ & & \\
\hline & 6 months-baseline & $2.4(50.8, n=153)$ & $0.5(49.5, n=177)$ & & \\
\hline & $\dagger^{*}$ Baseline & $108.1(71.9, n=98)$ & $98.6(61.1, n=115)$ & $-0.55(-13.9$ to 12.8$)$ & 0.935 \\
\hline & 12 months & $99.4(72.8, n=98)$ & $87.6(62.3, n=115)$ & & \\
\hline & 12 months-baseline & $-8.7(53.0, n=98)$ & $-11.0(48.5, n=115)$ & & \\
\hline \multirow{6}{*}{$\begin{array}{l}\text { PDQ-39 } \\
\text { (0-100, higher values worse) }\end{array}$} & tBaseline & $27.4(14.3, n=126)$ & $28.7(15.9, n=153)$ & 0.12 ( -2.0 to 2.28$)$ & 0.911 \\
\hline & 6 months & $28.3(15.0, n=126)$ & $29.5(16.5, n=153)$ & & \\
\hline & 6 months-baseline & $0.8(8.3, n=126)$ & $0.9(9.0, n=153)$ & & \\
\hline & $\dagger *$ Baseline & $27.2(13.6, n=77)$ & $28.9(15.9, n=100)$ & $0.48(-2.53$ to 3.49$)$ & 0.754 \\
\hline & 12 months & $29.1(15.4, n=77)$ & $31.7(15.5, \mathrm{n}=100)$ & & \\
\hline & 12 months-baseline & $1.9(8.6, n=77)$ & $2.8(11.2, n=100)$ & & \\
\hline \multirow{6}{*}{$\begin{array}{l}\text { GDS } \\
\text { (0-15, higher values worse) }\end{array}$} & tBaseline & $7.7(2.3, n=154)$ & $7.7(2.1, n=183)$ & $-0.02(-0.42$ to 0.39$)$ & 0.942 \\
\hline & 6 months & $7.8(2.5, \mathrm{n}=154)$ & $8.0(2.5, n=183)$ & & \\
\hline & 6 months-baseline & $0.3(1.8, n=154)$ & $0.2(1.9, n=183)$ & & \\
\hline & $\dagger *$ Baseline & $7.7(2.1, n=96)$ & $8.0(2.2, n=118)$ & $-0.21(-0.72$ to 0.31$)$ & 0.421 \\
\hline & 12 months & $7.8(2.5, \mathrm{n}=96)$ & $8.5(2.4, n=118)$ & & \\
\hline & 12 months-baseline & $0.2(2.0, n=96)$ & $0.4(1.7, n=118)$ & & \\
\hline
\end{tabular}

${ }^{*}$ Controlled for site, age, gender, repeat falling or not in the year prior to screening, log number of falls in the year prior to falling, log rate of falling in the prerandomisation falls collection period, Hoehn and Yahr and the outcome in question assessed at baseline.

†Baseline results restricted to existing participant assessment at 6 months.

FES-I, Falls Efficacy Scale International; GDS, Geriatric Depression Scale; PASE, Physical Activity Scale for the Elderly; PDQ-39, Parkinson's Disease Questionnaire. 


\begin{tabular}{|c|c|c|c|c|c|}
\hline & & & $\begin{array}{l}\text { PDSAFE } \\
(n=238)\end{array}$ & $\begin{array}{l}\text { Control } \\
(\mathrm{n}=236)\end{array}$ & $P$ value* \\
\hline \multirow{2}{*}{$\begin{array}{l}\text { Baseline among participants with } \\
6 \text {-month assessment }\end{array}$} & \multicolumn{2}{|c|}{ Unable of those with a CST assessment } & $29 / 188(15 \%)$ & $35 / 213(16 \%)$ & \multirow{4}{*}{$\begin{array}{l}0.041 \\
(\mathrm{n}=401)\end{array}$} \\
\hline & CST (s) among those able & $\begin{array}{l}\text { Median } \\
\text { IQR }\end{array}$ & $\begin{array}{l}14 \\
11-18 \\
(n=159)\end{array}$ & $\begin{array}{l}14 \\
11-18 \\
(n=178)\end{array}$ & \\
\hline \multirow[t]{2}{*}{ 6-month assessment } & \multicolumn{2}{|c|}{ Unable of those with a CST assessment } & $27 / 188(14 \%)$ & $47 / 213(22 \%)$ & \\
\hline & CST (s) among those able & $\begin{array}{l}\text { Median } \\
\text { IQR }\end{array}$ & $\begin{array}{l}12 \\
10-15 \\
(n=161)\end{array}$ & $\begin{array}{l}13 \\
10-16 \\
(n=166)\end{array}$ & \\
\hline \multirow{2}{*}{$\begin{array}{l}\text { Baseline among participants with } \\
12 \text {-month assessment }\end{array}$} & \multicolumn{2}{|c|}{ Unable of those with a CST assessment } & $15 / 119(13 \%)$ & $21 / 134(16 \%)$ & \multirow{4}{*}{$\begin{array}{l}0.163 \\
(n=253)\end{array}$} \\
\hline & CST (s) among those able & $\begin{array}{l}\text { Median } \\
\text { IQR }\end{array}$ & $\begin{array}{l}14 \\
11-17 \\
(n=104)\end{array}$ & $\begin{array}{l}14 \\
12-18 \\
(n=113)\end{array}$ & \\
\hline \multirow[t]{2}{*}{ 12-month assessment } & \multicolumn{2}{|c|}{ Unable of those with a CST assessment } & $23 / 119(19 \%)$ & $38 / 134(28 \%)$ & \\
\hline & CST (s) among those able & $\begin{array}{l}\text { Median } \\
\text { IQR }\end{array}$ & $\begin{array}{l}12 \\
9-14 \\
(n=96)\end{array}$ & $\begin{array}{l}13 \\
11-15 \\
(n=96)\end{array}$ & \\
\hline
\end{tabular}

* Mann-Whitney U test incorporating participants unable to perform test and times from participants able to perform test.

tai chi. ${ }^{32}$ A meta-analysis of fall interventions prior to this trial demonstrated an inconsistency of effect with a need for higher quality trials. $^{2}$ In line with those previous researchers of non-significant trials, we found a diverse response to the intervention, those with milder disease responding better to the intervention than those with a more severe condition profile.

It has been suggested that non-significant fall prevention trials have resulted from underdosing of treatment ${ }^{3}$ but dosing rehabilitation is complex. Rehabilitation is an interactive educational process designed to improve function and facilitated by a therapist. Each person has to actively participate in rehabilitation, the process of which can be influenced by motivation, age, cognition and severity of condition. Other important features are timing and content of intervention as well as the therapists and training. We will address these issues below and discuss how they may have led to the non-significant finding of our trial.

First, the influence of the therapy content and delivery on rehabilitation dose. A strength of the trial was the evidencebased therapy programme delivered by skilled trained physiotherapists. The PDSAFE training was structured and practice was

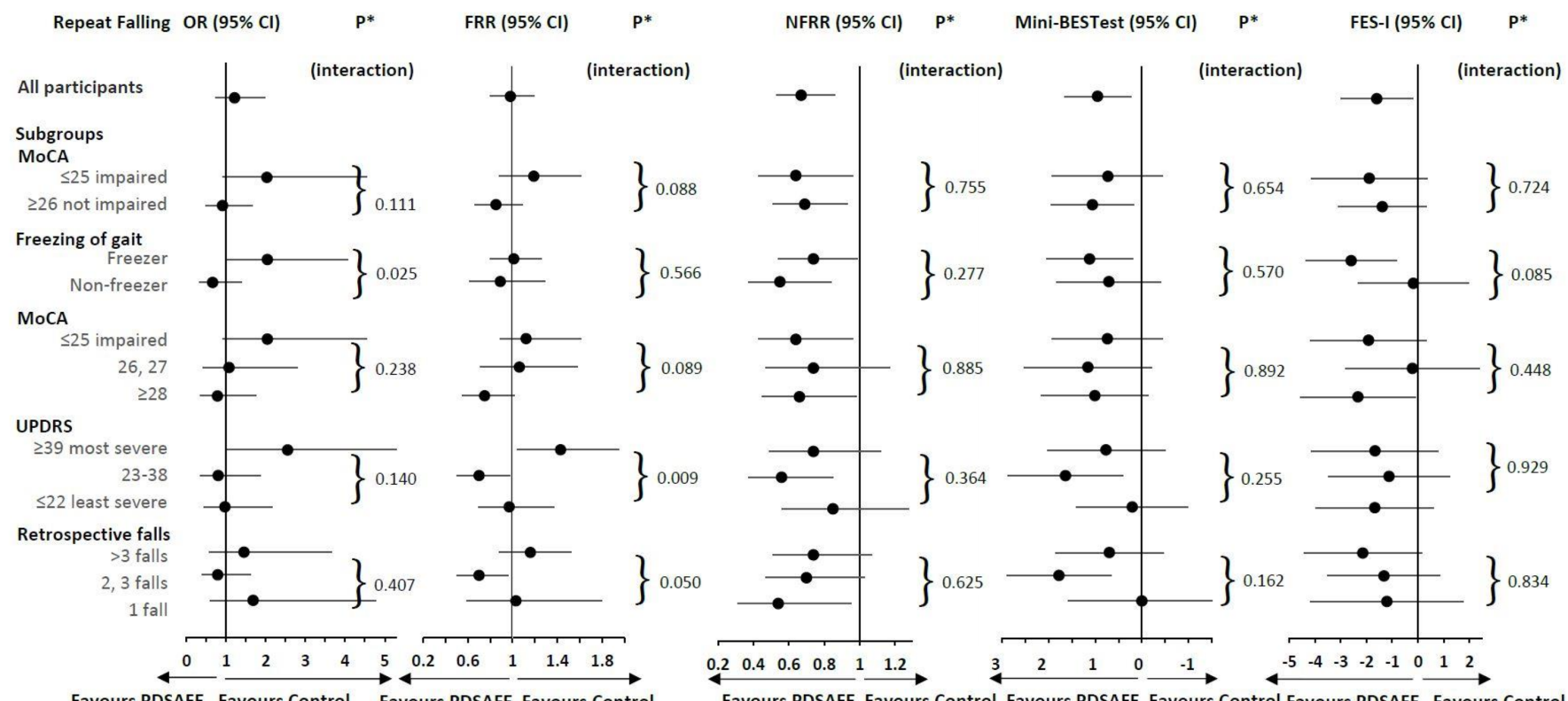

* Test for interaction of PDSAFE contrast differing across subgroups, controlled for site, age, gender, repeat falling or not in the year prior to screening, log number of falls in the year prior to falling, log rate of falling in the pre randomisation falls collection period, Hoehn and Yahr, and the outcome in question assessed at baseline.

**OR= Odds Ratio; FRR=Falls Rate Ratio; NFRR= Near Falls Rate Ratio; Mini-BESTest= Balance Evaluation Systems Test; FES-I= Falls Efficacy Scale International

Figure 2 Overall and subgroup analysis of falling, near falling (during 0-6 months) and secondary outcomes (at 6 months). FES-I, Falls Efficacy Scale International; FRR, fall rate ratio; Mini-BESTest, balance evaluation systems test; MoCA, Montreal Cognitive Assessment; NFRR, near fall rate ratio; UPDRS, Unified Parkinson's Disease Rating Scale. 
rigorously tested for fidelity. Ninety-one per cent of the intervention group participants received per protocol on average 12 faceto-face supervised sessions of 1-1.5 hours and were encouraged to complete unsupervised daily practice, meeting the recommendations of Sherrington et $a l^{33}$ of at least 50 hours of practice. Previous researchers have used a range of different interventions, often poorly described with a great deal of diversity of treatment protocols and dosage. The PDSAFE trial delivered more supervised sessions than any of the other five identified research trials, for example, 1 hour weekly face to face for 6 weeks, ${ }^{5} 1$ hour weekly group session for 8 weeks, ${ }^{6} 1$ hour weekly group session for 10 weeks, ${ }^{7} 1$ hour monthly group session for 6 months and 1 hour weekly session for 6 weeks $^{8}$; in addition, all encouraged independent practice but their recommendations were not as frequent as the PDSAFE mandate of daily routine. In terms of amount of treatment a balance is needed between sufficient time for training and too high an expectation of commitment, if too high participants start to withdraw and even under our procedures we had a greater withdrawal from the intervention group than our control group. Our programme was personalised in order to maximise individual motivation and it was multidimensional, requiring individuals to progress their activities and to integrate their training into everyday functional tasks which needed active participation from them and cognitive reasoning but this may have been too challenging for some. While manageable by those in the moderate to least severe disease groups, attention, reaction and response rates may have been compromised at the more severe end of the spectrum even though the treatment and selection of tasks were personalised.

Second, the influence of individual characteristics on rehabilitation dose. Our sample reflected a typical heterogeneous Parkinson's population and was not dissimilar to the other research trials; it comprised participants with a range of disease severity and fall events; at least one fall in the previous 12 months, some with multiple falls and high disease severity or more complex symptoms, such as freezing. Within Parkinson's, falls are indicative of a changing disease process; PwP with an established pattern of falling will have more global features of decline of age and/or disease association. Lord et $a l^{34}$ suggest these progressive features create additional challenges for implementing appropriate management and research. Our sample typically included those with and without freezing of gait and, although we screened to exclude individuals with cognitive impairment using the MMSE, ${ }^{17}$ some participants at baseline and subsequent assessments scored low on the MoCA, ${ }^{28}$ a test known to be sensitive to frontal and executive function associated with people who fall. ${ }^{35}$ The motor learning potential is reduced in people who freeze ${ }^{3637}$ and are cognitively challenged; increasing evidence demonstrates that freezing of gait is an important risk factor of falls, associated with reduced balance ${ }^{38}$ and impaired cognition. The inclusion of people who did or did not experience freezing illustrates the diverse range of motor problems present in a community sample of PwP and the range of challenges faced when teaching and learning new skills. Domingos et $a l^{39}$ have called for more studies into fall prevention with people with impaired cognition.

That exercise is not uniformly effective across all presentation of Parkinson's had been suggested but not confirmed in three previous smaller trials, ${ }^{6-8}$ this informed our decision to prespecify and undertake subgroup analyses on our data. The recent work of Canning et $a l^{6}$ and older trials by Goodwin $e t a l^{7}$ and Ashburn et $a l^{8}$ consistently found in their secondary analysis that those with less severe Parkinson's responded positively to fall reduction programmes but that effect had been counterbalanced with deterioration among those at the worse end of the spectrum producing a non-significant overall result. Although we have a number of subgroup analyses in PDSAFE, the results are consistent with other published estimates reflecting a similar pattern: those in the moderate disease group, and in the middle group with respect to number of falls prior to the trial had a positive effect from PDSAFE with a 30\% reduction in FRR (95\% CI 50\% to $5 \%$ reduction) in the first 6 months following randomisation. However, there was an increase in FRR of 43\% (95\% CI 4\% to 95\%) helpful for the moderate disease group, more or different supervised therapy may have been required for those with freezing of gait and greater disease severity (more likely to have additional cognitive impairment) as they could have been more challenged by fall avoidance strategies and experienced greater difficulty with engaging in unsupervised sessions, thus limiting the intensity of practice and the effects.

A number of previous trials ${ }^{2}$ have shown the benefits of exercise on risk factors for falling. In PDSAFE balance, functional strength and falls efficacy were targeted in the treatment and our analysis of secondary outcomes demonstrated a consistent improvement across the whole PDSAFE group, that is, those with mild, moderate and severe disease. These results support those of others ${ }^{2}$ highlighting the positive effects of exercise for balance and gait ability. Interventions in previous trials have largely been delivered outside the home setting but our current results demonstrate similar findings from interventions within the home environment. A positive PDSAFE effect on near falls, an indicator of instability and reflective of the improvements in balance, was also reported. Near falls among PwP have been reported by Gazibara et $a l^{40}$ and Ashburn et $a l^{8}$ and are often related to stumbling or balance loss and have been observed to predispose falling in the general older population. ${ }^{41}$ As previously highlighted, all participants, even those with cognitive impairments, greater disease severity and freezing of gait, showed improvements in their balance scores and FES. We suggest this indicates these participants were able to comply with exercise but were not able to benefit from the more demanding fall prevention programme possibly because of the need to react and respond in a timely way at moments of instability.

A limitation of our research was the incomplete 12-month assessment on our sample, a consequence of restricted funding. All participants in our trial were followed to 6 months, the point of primary outcome, but the last 132 recruits could not be followed to 1 year after randomisation resulting in a smaller sample at 12 months impacting on the interpretation of longterm follow-up, including the economic evaluation.

\section{CONCLUSION}

PDSAFE did not reduce falls in the overall heterogeneous sample of PwP. The treatments were personalised to individual needs within the structure of the PDSAFE concept but the package may not have adequately addressed the range of profiles across the disease spectrum. Those with more severe disease may have found implementing the fall avoidance movement strategies too challenging or they needed more training time; secondary analysis showed diverse responses to the intervention. Secondary outcome measures demonstrated improvement in balance, functional strength and falls efficacy with a reduction in near falls across the whole sample. Further confirmatory trials of fall prevention on targeted groups of PwP are recommended. version 24 
Acknowledgements We acknowledge and thank the participants with Parkinson's; PPI representatives; and members of the Steering and Data Monitoring Committee for their valued contributions to the trial.

Contributors $K S$ and $A A$ led the trial and drafted the manuscript. $A A, R P, E M, L R$, $H R, V G, A N, C B$ and SEL were grant holders and each contributed to study design and management, data interpretation and critical reviewing. $A A, S H, L R, A N$ and VG designed the intervention. RP and IRM provided statistical expertise including data cleaning, analysis and interpretation, and writing. CF, SH and DK contributed to data collection, fidelity checking, data cleaning and data interpretation.

Funding The project was funded by the National Institute for Health Research (NIHR) HTA programme (project number 10/57/21). SEL is funded by the NIHR Collaboration for Leadership in Applied Health Research and Care at Oxford Health NHS Trust and the Oxford Biomedical Research Centre at the Oxford University Hospital Trust, and CLAHRC Oxford. VG is supported by the NIHR Collaboration for Leadership in Applied Health Research and Care in the South West Peninsula (PenCLAHRC). LR is supported by the NIHR Newcastle Biomedical Research Centre (BRC) based at Newcastle upon Tyne Hospitals NHS Foundation Trust and Newcastle University. The research was also supported by NIHR Newcastle CRF Infrastructure funding. HR is supported by CLAHRC Wessex and NIHR Southampton Biomedical Research Centre.

Disclaimer The views and opinions expressed therein are those of the authors and do not necessarily reflect those of the HTA programme, NIHR, NHS or the Department of Health.

Competing interests $L R$ reports grants from Newcastle University during the conduct of the study; grants from Parkinson's UK, grants from EU Marie Curie Training Network, grants from MRC, grants from EPSRC, grants from Wellcome Trust, from Stroke Association, outside the submitted work. CB is a member of the Primary Care Community and Preventive Interventions HTA group and the associated Methods group. VG reports grants from the National Institute for Health Research, during the conduct of the study. SEL reports grants from the NIHR Health Technology Assessment Programme during the conduct of this study. SEL was a member of the HTA Additional Capacity Funding Board, HTA End of Life Care and Add-on Studies, HTA Prioritisation Group, HTA Trauma Board during this study. All other authors have nothing to disclose.

Patient consent Not required.

Ethics approval Ethics approval was granted by the NRES South Central Hampshire B Committee (REC reference 14/SC/0039).

Provenance and peer review Not commissioned; externally peer reviewed.

Open access This is an open access article distributed in accordance with the Creative Commons Attribution Non Commercial (CC BY-NC 4.0) license, which permits others to distribute, remix, adapt, build upon this work non-commercially, and license their derivative works on different terms, provided the original work is properly cited, appropriate credit is given, any changes made indicated, and the use is non-commercial. See: http://creativecommons.org/licenses/by-nc/4.0/.

\section{REFERENCES}

1 Gillespie LD, Robertson MC, Gillespie WJ, et al. Interventions for preventing falls in older people living in the community. Cochrane Database Syst Rev 2012;(9):CD007146

2 Shen X, Wong-Yu ISK, Mak MKY. Effects of Exercise on Falls, Balance, and Gait Ability in Parkinson's Disease

3 Morris ME, Menz HB, McGinley JL, et al. A Randomized Controlled Trial to Reduce Falls in People With Parkinson's Disease. Neurorehabil Neural Repair 2015;29:777-85.

4 Mirelman A, Rochester L, Maidan I, et al. Addition of a non-immersive virtual reality component to treadmill training to reduce fall risk in older adults (V-TIME): a randomised controlled trial. Lancet 2016;388:1170-82.

5 Morris ME, Taylor NF, Watts JJ, et al. A home program of strength training, movement strategy training and education did not prevent falls in people with Parkinson's disease: a randomised trial. J Physiotherapy 2017;63:94-100.

6 Canning C, Sherrington C, Lord S, et al. Fung V Exercise for falls prevention in Parkinson's disease. Neurology 2015:84:304-12.

7 Goodwin VA, Richards SH, Henley W, et al. An exercise intervention to prevent falls in people with Parkinson's disease: a pragmatic randomised controlled trial. J Neuro Neurosurg \& Psych 2011;82:1232-8.

8 Ashburn A, Fazakarley L, Ballinger C, et al. A randomised controlled trial of an exercise programme for people with Parkinson's disease who repeatedly fall. J Neuro Neurosurg Psych 2007;78:678-84.

9 Pickering RM, Grimbergen YAM, Rigney U, et al. A meta-analysis of six prospective studies of falling in Parkinson's disease. Mov Disord. 2007;22:1892-900.

10 Canning CG, Paul SS, Nieuwboer A. Prevention of falls in Parkinson's disease: a review of fall risk factors and the role of physical interventions. Neurodegenerative Disease Management 2014:4:203-21.
11 van der Marck MA, Klok MPC, Okun MS, et al. Consensus-based clinical practice recommendations for the examination and management of falls in patients with Parkinson's disease. Parkinsonism Relat Disord 2014;20:360-9.

12 Bekkers EMJ, Dijkstra BW, Dockx K, et al. Clinical balance scales indicate worse postural control in people with Parkinson's disease who exhibit freezing of gait compared to those who do not: A meta-analysis. Gait Posture 2017:56:134-40.

13 Fasano A, Canning CG, Hausdorff JM, et al. Falls in Parkinson's disease: a complex and evolving picture. Movement Disorders 2017;32:1524-36.

14 Muzerengi S, Herd C, Rick C, et al. A systematic review of interventions to reduce hospitalisation in Parkinson's disease. Parkinsonism Relat Disord 2016;24(Supplement C):3-7.

15 Goodwin VA, Pickering R, Ballinger $C$, et al. A multi-centre, randomised controlled tria of the effectiveness of PDSAFE to prevent falls among people with Parkinson's: study protocol. BMC Neurology 2015:15.

16 National Institute for Health and Clinical Excellence. Parkinson's Disease: Diagnosis and management in primary \& secondary care. London: Royal College of Physicians, 2006 Report No. CG35.

17 Folstein MF, Folstein SE, McHugh PR, et al. A practical method for grading the cognitive state of patients for the clinician. J Psych Res 1975;12:189-98.

18 Hulbert S, Rochester L, Nieuwboer A, et al. "Staying safe" - a narrative review of falls prevention in people with Parkinson's - "PDSAFE". Disabil Rehabil.

19 Hauer K, Lamb SE, Jorstad EC, et al. Systematic review of definitions and methods of measuring falls in randomised controlled fall prevention trials. Age Ageing 2006;35:5-10

20 King L, Horak F. On the Mini-BESTest: scoring and the reporting of total scores. Physical Therapy 2013;93:571-5

21 Duncan RP, Leddy AL, Earhart GM. Five times sit-to-stand test performance in Parkinson's disease. Arch Phys Med Rehabil 2011;92:1431-6.

22 Yesavage JA, Scale GD. Geriatric depression scale. Psychopharmacol Bull 1988;24:709-11.

23 Yardley L, Beyer N, Hauer K, et al. Development and initial validation of the falls efficacy Scale-International (FES-I). Age and Ageing 2005;34:614-9.

24 Nieuwboer A, Rochester L, Herman T, et al. Reliability of the new freezing of gait questionnaire: agreement between patients with Parkinson's disease and their carers. Gait Posture 2009:30:459-63.

25 Brown RG, MacCarthy B, Jahanshahi $M$, et al. Accuracy of self-reported disability in patients with parkinsonism. Arch Neurol 1989;46:955-9.

26 Washburn RA, Smith KW, Jette AM, et al. The physical activity scale for the elderly (PASE): development and evaluation. J Clin Epidemiol 1993;46:153-62.

27 EuroQol - a new facility for the measurement of health-related quality of life. Health Policy 1990;16:199-208. 01-12-1990.

28 Nasreddine ZS, Phillips NA, Bedirian V, et al. The Montreal Cognitive assessment, MoCA: a brief screening tool for mild cognitive impairment. J Am Geriatr Soc 2005:53:695-9.

29 Hoehn M, Parkinsonism YM. Onset, progression and mortality. Neurol 1967:17:1427-42.

30 Lang A, Fahn S. Assessment of Parkinson's Disease. In: Munsat T, ed. Quantification of neurologic deficit, chapter 21. MA; Butterworths, 1989.

31 StataCorp. Stata statistical software: release 11. College Station, TX: StataCorp LP., 2009

32 Li F, Harmer P, Fitzgerald K, et al. Tai chi and postural stability in patients with Parkinson's disease. N Engl J Med 2012:366:511-9.

33 Sherrington C, Whitney JC, Lord SR, et al. Effective exercise for the prevention of falls: a systematic review and meta-analysis. JAM Geriatr Soc 2008;56:2234-43.

34 Lord S, Galna B, Yarnall AJ, et al. Natural history of falls in an incident cohort of Parkinson's disease: early evolution, risk and protective features. J Neurol 2017:264:2268-76.

35 Zadikoff C, Fox SH, Tang-Wai DF, et al. A comparison of the mini mental state Exam to the Montreal Cognitive assessment in identifying cognitive deficits in Parkinson's disease. Movement Disorders 2008:23:297-9.

36 Heremans E, Nackaerts E, Vervoort G, et al. Impaired retention of motor learning of writing skills in patients with Parkinson's Disease with freezing of gait. Plos One 2016:11:e0148933.

37 Heremans E, Nieuwboer A, Spildooren J, et al. Cognitive aspects of freezing of gait in Parkinson's disease: a challenge for rehabilitation. J Neural Transm 2013;120:543-57

38 Allen NE, Schwarzel AK, Canning CG. Recurrent Falls in Parkinson's Disease: A Systematic Review. Parkinsons Dis 2013;2013:1-16.

39 Domingos JM, Godinho C, Dean J, et al. Cognitive impairment in fall-related studies in Parkinson's disease. J Parkinsons Dis 2015;5:453-69.

40 Gazibara T, Kisic Tepavcevic D, Svetel M, et al. Near-falls in people with Parkinson's disease: circumstances, contributing factors and association with falling. Clin Neurol Neurosurg 2017;161:51-5.

41 Srygley JM, Herman T, Giladi N, et al. Self-report of missteps in older adults: a valid proxy of fall risk? Arch Phys Med Rehabil 2009:90:786-92. 